\section{Is Dexmedetomidine a Favorable Agent for Cerebral Hemodynamics?}

Received: October 12, 2015; Accepted: October 15, 2015; Published: October 22, 2015

Hemodynamic stability, with special attention to arterial pressure in order to warrant an adequate cerebral perfusion, is a cornerstone of Neurocritical care (NCC) management. An abrupt elevation of arterial blood pressure can aggravate cerebral edema or induce cerebral hematoma, resulting in a prolonged NCC unit stay, on the other hand, hypotension is associated with an increased risk for cerebral ischemia that is more pronounced when auto regulation of cerebral blood flow (CBF) is impaired and there is a compromised cerebral compliance $[1,2]$. Moreover, any derangement of cerebrovascular hemodynamics may contribute to intracranial pressure (ICP) elevation with concomitant cerebral perfusion pressure (CPP) deterioration, which can further exacerbate ischemic damage [3].

Thus, the ideal sedative agent in NCC setting should have minimal impact on hemodynamics. Dexmedetomidine (Dex), a highly selective a2-adrenoreceptor agonist is emerging as a potentially attractive adjunct in the neurosurgical care due to its pharmacology promise benefits [4]. Nonetheless, the appealing performance of Dex in NCC practice is tempered by the reported unfavorable hemodynamic sequelae, consisting of bradycardia, hypotension, and hypertension. The biphasic effect of Dex on blood pressure, with hypotension following the transient hypertension, is a well-described phenomenon [2].

Of utmost interest in NCC is also the impact of Dex on cerebral blood flow (CBF) and metabolism $\left(\mathrm{CMRO}_{2}\right)$ coupling. Existing evidence supports a dose-dependent decline in CBF after Dex administration secondary to cerebrovascular vasoconstriction, being almost $30 \%$ at clinically relevant Dex concentrations [4]. It seems that, the clinical impact of CBF deterioration is directed by the clinical circumstances. The use of, Dex might be useful adjunct in clinical situations when an increase in CBF could be detrimental such as vasogenic cerebral oedema (i.e., traumatic brain injury, large brain tumors). Nevertheless, Dex infusion is questionable in patients with subarachnoid hemorrhage $(\mathrm{SAH})$ and acute stroke, since the associate drop in arterial pressure could worsen the coexisting increase in circulating catecholamines and massive sympathetic outflow. Although, evidence from experimental studies shows that $\mathrm{CMRO}_{2}$ remains unaffected by the use of Dex, no relevant data from human studies exist [4]. Despite the reported neuroprotective effects of Dex in models of ischemic brain injury, the aforementioned clinical features raise concerns that reduction of CBF in the face of an unaltered reduction of $\mathrm{CMRO}_{2}$, potentially limits adequate cerebral

\section{Georgia Tsaousi ${ }^{1}$ and Federico Bilotta ${ }^{2}$}

1 Department of Anesthesiology and ICU, Faculty of Medicine, Aristotle University of Thessaloniki, Greece

2 Department of Anesthesiology, University of Rome "La Sapienza”, Rome, Italy

Corresponding author: Georgia Tsaousi

tsaousig@otenet.gr

Anesthetist-Intensivist, Assistant Professor of Anesthesiology, Faculty of Medicine, Aristotle University of Thessaloniki, Maiandrou 32, GR 56224 Thessaloniki Greece

Tel: +302310994855

Fax: 302310994860

Citation: Tsaousi G, Bilotta F.

Is Dexmedetomidine a Favorable Agent for Cerebral Hemodynamics?. J Intensive \& Crit Care 2015, 1:1.

oxygenation of brain tissue at risk for ischemic injury. The clinical significance of these effects is uncertain at the present time.

The last key-element of cerebral hemodynamics is ICP, as the elevated ICP in NCC setting promotes CBF deterioration, thus leading to a potential global or regional cerebral ischemia. The extremely limited clinical data, however, failed to confirm a notable alteration of ICP during Dex administration [5].

Much of the knowledge accumulated on the use of Dex in the NCC setting is derived by limited quality evidence. The use of Dex is debatable, as NCC encompasses subgroups of patients such as TBI, SAH and intracerebral hemorrhage ones in whom there is the unique need to maintain supranormal blood pressure values with a view to ensure adequate cerebral perfusion and to optimize outcome $[1,3]$. Considering that, the primary indication for Dex use was as an adjunctive sedative in intensive care unit (ICU) setting and the numerous reports evaluating the use of Dex in general ICU setting for the last two decades, the existing evidence for Dex use in NCC is extremely limited, including mainly observational trials with variable methodological quality, non- 
consistent design and target population. These few studies dealing with the efficacy and safety of Dex as sole agent or an adjunct to traditional sedatives, seem to be inconclusive $[2,6,7]$. Nevertheless, these reports assess only short-term outcomes, and no information about long-term impact regarding Dex use in NCC is provided.

In conclusion, available clinical evidence on the use of Dex in NCC is limited, and no definite conclusion can be drawn until more rigorously designated trials elucidate the effect of Dex administration as a sole sedative agent or as adjunct to other sedatives on systemic and cerebral hemodynamics and its impact on short-term and long-term outcomes in various NCC subpopulations. According available evidence, there are possible warnings on the use of Dex in NCC due to associated hemodynamic effects -especially those related to reduction of arterial pressurethat might ultimately lead to suboptimal cerebral perfusion. 


\section{References}

1 Basali A, Mascha EJ, Kalfas I, et al. (2000) Relation between perioperative hypertension and intracranial hemorrhage after craniotomy. Anesthesiology 93: 48-54.

2 Wang X, Ji J, Fen L, Wang A (2013) Effects of dexmedetomidine on cerebral blood flow in critically ill patients with or without traumatic brain injury: a prospective controlled trial. Brain Inj 27: 1617-1622.

3 Peng K, Wu S, Liu H, Ji F (2014) Dexmedetomidine as an anesthetic adjuvant for intracranial procedures: meta-analysis of randomized controlled trials. J Clin Neurosci 21: 1951-1958.
4 Grewal A (2011) Dexmedetomidine: New avenues. J Anaesthesiol Clin Pharmacol 27: 297-302.

5 Talke P, Tong C, Lee HW, Caldwell J, Eisenach JC, et al. (1997) Effect of dexmedetomidine on lumbar cerebrospinal fluid pressure in humans. Anesth Analg 85: 358-364.

6 Srivastava VK, Agrawal S, Kumar S, Mishra A, Sharma S, et al. (2014) Comparison of dexmedetomidine, propofol and midazolam for short-term sedation in postoperatively mechanically ventilated neurosurgical patients. J Clin Diagn Res 8: GC04-7.

7 Erdman MJ, Doepker BA, Gerlach AT, Phillips GS, Elijovich L, et al. (2014) A comparison of severe hemodynamic disturbances between dexmedetomidine and propofol for sedation in neurocritical care patients. Crit Care Med 42: 1696-1702. 